\title{
Natural history of non cirrhotic portal fibrosis - a tropical experience
}

\begin{abstract}
Non cirrhotic portal fibrosis is a clinical entity of uncertain etiology. The disease is usually non progressive, though a subset of patients may progress to progressive liver failure. Various factors have been implicated in the causation of the disease. The present review highlights the natural history of this entity.
\end{abstract}

Volume 6 Issue 2 - 2017

\author{
Jayanthi V, Mayank Jain, Mukul Vij, Joy \\ Varghese
}

Institute of Liver Disease and Liver Transplant, India

Correspondence: V Jayanthi, Global Health City, Chennai, India, 600020, India, Email drjayanthi35@yahoo.co.in

Received: October 27, 2016 | Published: February 09, 2017

\section{Introduction}

Non-Cirrhotic Portal Hypertension (NCPH) includes Extra Hepatic Portal Vein Obstruction (EHPVO) and Non-Cirrhotic Portal Fibrosis (NCPF). The natural history of NCPH especially NCPF is not clear. Non-cirrhotic portal fibrosis (NCPF) is a clinical entity of uncertain etiology that is typically characterized by periportal fibrosis due to involvement of small and medium branches of the portal vein, resulting in the portal hypertension. ${ }^{1}$ In Japan this is referred to as idiopathic portal hypertension. In rest of the of the world it has been named as hepatoportal sclerosis, ${ }^{2,3}$ non cirrhotic intrahepatic portal hypertension (NCIPH $)^{4}$ and idiopathic non cirrhotic intrahepatic portal hypertension (INIPH). ${ }^{5}$ From Southern India, the same clinical entity is being described as non cirrhotic idiopathic portal hypertension (NCIPH) and is considered as an important cause for cryptogenic cirrhosis with confirmation on liver biopsy. ${ }^{6,7}$

In India, in the early 1990's, NCPF was an important cause for $\mathrm{NCPH}$ and was increasingly reported from the northern parts of Indian subcontinent and constituted $15-30 \%$ of all causes of presinusoidal portal hypertension [8,9]. However, its incidence has decreased considerably over a period of time. ${ }^{1}$ The present review highlights the literature focusing on the natural history of NCPF with personal observations made on this clinical entity during clinical practice.

\section{Clinico - pathological correlation in NCPF}

Clinically, patients with NCPF are young men in the third and fourth decade of life. , $^{810-12}$ They usually present with recurrent well tolerated upper gastrointestinal bleed, requiring multiple blood transfusions, compensated in most instances, with a mild to moderate hepatomegaly, a massive splenomegaly, anemia and thrombocytopenia. ${ }^{11}$ Approximately, $10 \%-34 \%$ do present with ascites. ${ }^{13}$ Death in these patients is often due to a variceal bleed rather than liver dysfunction.

\section{Natural history}

$\mathrm{NCPF}$ is often considered as non progressive, though 10\% may manifest present with ascites, an acute on chronic liver failure and even end stage liver disease. Survival is prolonged with complete asymptomatic intervals once variceal bleed is controlled by either endoscopic eradication of varices or reduction in portal pressure by a surgical shunt.

\section{Gross and histopathological description}

The spectrum of gross findings in NCPF ranges from those seen in EHPVO and that seen in early cirrhosis.

On gross pathological specimens: The liver is either normal sized, enlarged, or at times even shrunken. The surface is likewise either smooth, bossalated, wrinkled, or may show surface nodularity simulating cirrhosis. Fibrous thickening of capsule of the liver with increased vascularity is frequently seen. ${ }^{14-16}$ The most consistent finding in the portal venous system is the dilatation of the main portal vein with thick sclerosed walls, together with thrombosis in medium and small portal vein branches, referred to as obliterative portal venopathy. ${ }^{17}$

On histopathology: Typical findings within the intrahepatic portal venous system are :

Parenchymal changes: These include, thin incomplete periportal / perisinusoidal septal fibrosis, preserved lobular architecture and differential atrophy. ${ }^{18-20}$ Incomplete septal cirrhosis and nodular regenerative hyperplasia may also be associated with NCPF or idiopathic portal hypertension and may be the late manifestation of this clinical entity. ${ }^{21-23}$

Intrahepatic portal veins: Findings include sinusoidal dilatation ((megasinusoids), obliterative portal venopathy i.e. luminal narrowing or obliteration of small portal venous branches (third degree branches and thereafter) accompanied by dense deposit of elastic fibers, increase in number of portal vascular channels (portal angiomatosus simulating intrahepatic portal cavernoma) and dilated portal veins herniating into the surrounding parenchyma (paraportal shunt vessels).

Portal venous trunk: The major portal vein shows thickening of the wall, endophlebosclerosis, intraluminal thrombosis and even calcification. ${ }^{17}$

\section{Pathogenesis}

The aetiology remains elusive. Some of the aetiological factors incriminated include arsenicosis, ${ }^{24,25}$ immunological abnormalities ${ }^{26}$ imbalance of von Willebrand factor and ADAMTS13, ${ }^{27}$ schistosomal infection and $\mathrm{E}$ coli sensitisation. ${ }^{28,29}$ Pathogenetic mechanism is unclear. Wanless hypothesised that the obliteration of portal venules results in progressive distortion of intrahepatic circulation 
with subsequent parenchymal remodeling. ${ }^{19}$ There is atrophy of the hepatocytes in areas with reduced portal blood supply and compensatory hyperplasia of the hepatocytes in well perfused areas, similar to that seen in partial nodular hyperplasia and nodular regenerative hyperplasia variants of intrahepatic NCPH. ${ }^{20}$ Eapen and others in recent publications ${ }^{27}$ on NCIPH, have incriminated fall in von Willebrand factor and a corresponding increase in ADAMTS13 as one of the likely triggers towards endophlebitis, formation and progression of thrombosis within the intrahepatic portal venous radicles, ischemia to neighboring hepatocytes and further progression to fibro cirrhotic changes.

On follow up of these patients, there is increasing evidence that in 20 to $33 \%$ of cases, the liver undergoes slow parenchymal atrophy due to reduced blood supply to the periphery, nodular transformation of the liver with extensive subhepatic (insular fibrosis) and portal fibrosis. In addition, the lack of compensatory hepatic arterial changes results in worsening of ischemia and this further contributes to liver cell failure. ${ }^{30}$

\section{Clinico - pathological correlation}

A study from North India reported an overlap of clinical features of NCPF and cirrhosis in around 5\% of patients with idiopathic portal hypertension. These patients terminally, developed ascites, jaundice, and hepatic encephalopathy making it difficult at times to differentiate this from end stage liver disease due to cirrhosis. ${ }^{31}$ Ten patients with hepatitis $\mathrm{C}$ (5 patients), hepatitis B (one patient) or non alcoholic fatty liver disease (4 patients) had characteristic portal vein changes. The existence of two aetiological possibilities i.e. virus and NCPF in a given patient is likely to result in rapid progression to ESLD.

Histopathological description of explant livers in these patients had shown areas of closely placed nodular hyperplastic parenchyma separated by compressed hepatocytes, portal fibrosis, mega sinusoids and hepatic peliosis. The major portal vein and its medium sized branches had variable grades of obliterative changes, the veins being replaced by fibrous intimal thickening with luminal compromise with or without thrombosis. ${ }^{32}$

Bernard et al. ${ }^{33}$ observed that few patients with idiopathic portal hypertension behaved like end-stage liver disease and developed features of decompensation in the form of ascites, jaundice and hepatic encephalopathy during the course of illness. In a subset of patients with NCPH, complications such as acute or chronic liver failure or an end stage liver disease with decompensation, ${ }^{34-39}$ and hepatopulmonary syndrome are severe enough to warrant a liver transplantation..$^{40,41}$ We had an opportunity to study, the gross and histology in liver explants (MJ) of 2 (both men) of 57 patients (3.5\%) with a pre - transplant diagnosis of cryptogenic cirrhosis but had NCPF in the explant specimen. Grossly, the liver (Figure 1) showed patchy surface nodularity. The cut surface showed vague nodulartity. The capsule was mild thickened. Light microscopy revealed variable portal expansion with dense fibroelastosis; fibrous septa were seen extending from the portal tracts into adjacent lobular parenchyma linking with other portal tracts (portal to portal fibrosis) and the central veins (portal to ventral vein). Crowding of the portal tracts was also appreciated. The portal veins show fibrointimal thickening, medial muscular hypertrophy and narrowed lumen in foci. Few aberrant blood vessels immediately adjacent to the peripheral portal tracts are noted. Obliterated portal veins with increased portal vascularity was also noted. The lobular parenchyma showed patchy macrovesicular steatosis along with mild ballooning and sinusoidal dilatation. The central vein also showed thickening of the wall in foci. Nodular regenerative hyperplasia was also noted (Figure 2). Thus features of NCPF were noted in both the liver explants of "cryptogenic cirrhosis" cases.

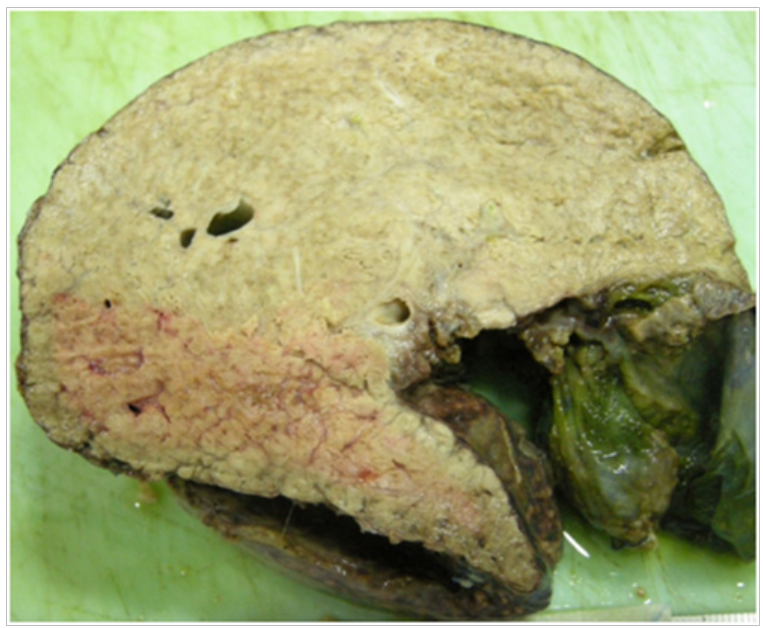

Figure I Gross image of an explant liver with NCPF.

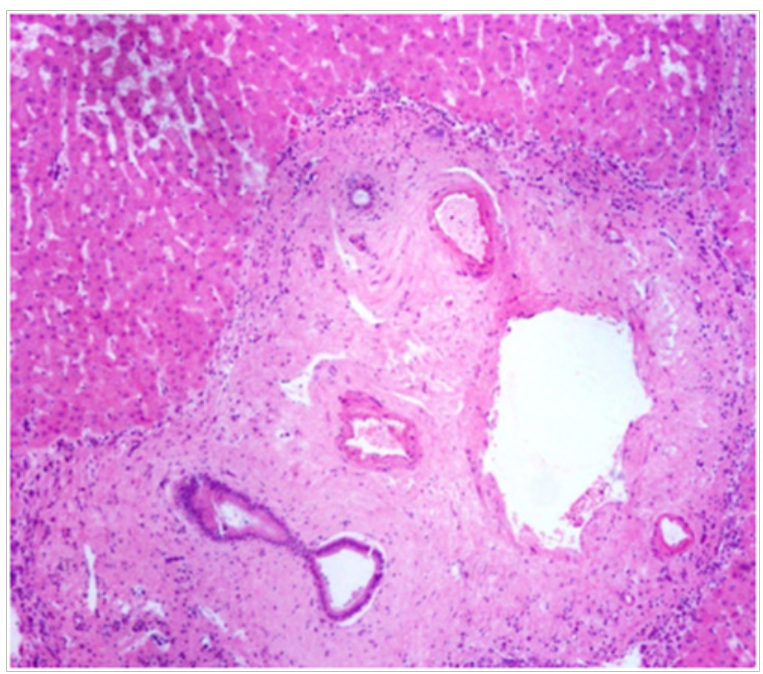

Figure 2 Light microscopy displaying dense portal fibrosis with portal vein thickening and luminal narrowing.

In a retrospective observational follow up study between 2001 and 2010, the senior author in their series of 34 patients (men age 24.9 years; male: female ratio $1: 1.8$ ) with a clinical, biochemical and ultrasonography features of NCPF, observed that at end of study, 20 $(58.82 \%)$ patients had no progression of stage of liver disease while $14(41.17 \%)$ progressed to cirrhosis over a mean period of 5.2 years. The longest follow up was for 10 years. Follow up with ultrasound and liver function tests at 6 monthly intervals, showed a significant reduction in size of the liver in 8 with differential enlargement of right and left lobes in the remaining 6. Eight patients developed ascites and required diuretics. These were patients who had classical and were followed up at 6 monthly intervals for progression of the problem until December 2010 [42]. Literature review and personal observations both clinically and in explant livers, has undoubtedly outlined that NCPF is indeed a dynamic state wherein there is progression of intrahepatic fibrosis secondary to phlebosclerosis of large and medium sized intrahepatic portal venous system; aetiology and pathogenesis remains speculative. 
In conclusion few important messages are forthcoming on natural history of NCPF:

i. Patients with NCPF have a wide spectrum of histopathological changes with varied clinical presentation that ranges from being asymptomatic to those presenting with recurrent upper gastrointestinal bleed, massive splenomegaly, cirrhosis, acute on chronic liver failure and even end stage liver disease with decomposition.

ii. NCPF seems to be a progressive clinical entity, wherein a proportion of patients do progress to cirrhosis over a period of time, some even requiring a liver transplant. What predisposes some to progress and not others is not clear. vWF deficiency and an increase in ADAMTS 13 as an aetiopathogenetic agent have been incriminated in Indian patients with NCPF.

iii. NCPF is likely to be one of the causes for cryptogenic cirrhosis. The latter two observations need prospective longitudinal studies for proving the hypothesis that NCPF is a progressive clinical entity from 'non cirrhotic' state to 'cirrhosis'. Future research into this direction would address some of our understanding and management of these patients with an 'idiopathic' diagnosis of liver cirrhosis.

\section{Acknowledgments}

None.

\section{Conflicts of interest}

The authors declare no conflict of interest.

\section{References}

1. Sarin SK, Kumar A, Chawla YK, et al. Noncirrhotic portal fibrosis/ idiopathic portal hypertension: APASL recommendations for diagnosis and treatment. Hepatol Int. 2007;1(3): 398-413.

2. Mikkelsen WP, Edmondson HA, Peters RL, et al. Extra- and intrahepatic portal hypertension without cirrhosis (hepatoportal sclerosis). Ann Surg. 1965;162(4): 602-620.

3. Bioulac-Sage, Le Bail B, Bernard PH, et al. Hepatoportal sclerosis. Semin Liver Dis. 1995;15(4): 329-39.

4. Kingham JGC, Levison DA, Stansfeld AG, et al. Non-cirrhotic intrahepatic portal hypertension: a long term follow-up study. $Q \mathrm{~J}$ Med. 1981;50(199):259-68

5. Hillaire S, Bonte $\mathrm{E}$, Denninger $\mathrm{MH}$, et al. Idiopathic non-cirrhotic intrahepatic portal hypertension in the West: a re-evaluation in 28 patients. Gut. 2002;51(2):275-280.

6. Madhu K, Avinash B, Ramakrishna B. Idiopathic non-cirrhotic intrahepatic portal hypertension: common cause of cryptogenic intrahepatic portal hypertension in a Southern Indian tertiary hospital. Indian J Gastroenterol. 2009;28(3):83-87.

7. Goel A, Madhu K, Zachariah U. A study of aetiology of portal hypertension in adults (including the elderly) at a tertiary centre in southern India. Indian J Med Res. 2013;137(5):922-927.

8. Bhargava DK, Dasarathy S, Sundaram KR, et al. Efficacy of endoscopic sclerotherapy on long-term management of esophageal varices: a comparative study of results in patients with cirrhosis of the liver, non-cirrhotic portal fibrosis (NCPF) and extra hepatic portal venous obstruction (EHO). J Gastroenterol Hepatol. 1991;6(5): 471-475.

9. Banghar PK, Abraham P, Mistry FP, et al. Profile of portal hypertension in Bombay. Indian J Gastroenterol. 1972;11(Suppl 1): A25.
10. Dhiman RK, Chawla Y, Vasishta RK, et al.Non-cirrhotic portal fibrosis (idiopathic portal hypertension): experience with 151 patients and a review of the literature. J Gastroenterol Hepatol. 2002;17(1): 6-16.

11. Pande C, Kumar A, Sarin SK. Non-cirrhotic portal fibrosis: a clinical profile of 366 patients. Am J Gastroenterol. 2006;101: S191.

12. Abraham P, Malkan GH, Bhatia SJ. Non-cirrhotic portal fibrosis in Bombay. Indian J Gastroenterol. 1995;14(Suppl 1): A97

13. Khanna R, Sarin S. Non-cirrhotic portal hypertension-Diagnosis and management. Journal of Hepatology. 2014;60 (2): 421-441

14. Okuda K, Nakashima T, Okudaira M, et al. Liver pathology of idiopathic portal hypertension. Comparison with non-cirrhotic portal fibrosis of India. The Japan idiopathic portal hypertension study. Liver. 1982;2(3):176-192.

15. Okudaira M, Ohbu M, Okuda K. Idiopathic portal hypertension and its pathology. Semin Liver Dis. 2002;22(1): 59-72.

16. Kage M, Arakawa M, Fukuda K, et al. Pathomorphologic study on the extrahepatic portal vein in idiopathic portal hypertension. Liver. 1990;10(4): 209-216.

17. Nayak NC, Ramalingaswami V. Obliterative portal venopathy of the liver. Associated with so-called idiopathic portal hypertension or tropical splenomegaly. Arch Pathol. 1969;87(4):359-369.

18. Hillaire $\mathrm{S}$, Bonte $\mathrm{E}$, Denninger $\mathrm{MH}$, et al. Idiopathic non-cirrhotic intrahepatic portal hypertension in the West: a re-evaluation in 28 patients. Gut. 2002;51(2): 275-280.

19. Wanless IR. Micronodular transformation (nodular regenerative hyperplasia) of the liver: a report of 64 cases among 2,500 autopsies and a new classification of benign hepatocellular nodules. Hepatology. 1990;11(5): 787-797.

20. Ludwig J, Hashimoto E, Obata H, et al. Idiopathic portal hypertension; a histopathological study of 26 Japanese cases. Histopathology. 1993;22(3): 227-234.

21. Bernard PH, Le Bail B, Cransac M. Progression from idiopathic portal hypertension to incomplete septal cirrhosis with liver failure requiring liver transplantation. J Hepatol. 1995;22(4):495-499.

22. Sciot R, Staessen D, Van Damme B (1988) Incomplete septal cirrhosis: histopathological aspects. Histopathology 13(6): 593-603.

23. Krasinskas AM, Eghtesad B, Kamath PS, Demetris AJ, Abraham SC (2005) Liver transplantation for severe intrahepatic noncirrhotic portal hypertension. Liver Transpl 11(6): 627-634.

24. Guha Mazumdar DN, Gupta JD, et al. Arsenic and non cirrhotic portal hypertension. J Hepatol. 1991;13: 376.

25. Santra A, Das Gupta JD, De B, et al.Hepatic manifestations in chronic arsenic toxicity. Indian J Gastroenterol. 1999;18(4):152-155.

26. Tokushige K, Yamauchi K, Komatsu T, et al. Predominant T helper 1 cells in patients with idiopathic portal hypertension. $J$ Gastroenterol Hepatol. 2000;15(11): 1312-1317.

27. Goel A, Alagammai PL, Nair SC. ADAMTS13 deficiency, despite well-compensated liver functions in patients with noncirrhotic portal hypertension. Indian J Gastroenterol. 2014;33(4): 355-363.

28. Kono K, Ohnishi K, Omata M, et al. Experimental portal fibrosis produced by intraportal injection of killed nonpathogenic Escherichia coli in rabbits. Gastroenterology. 1988;94(3):787-796.

29. Shekhar KC, Pathmanathan R. Hepatosplenic lesions induced by experimental Schistosoma malayensis in rabbits. Southeast Asian J Trop Med Public Health. 1993;24(2): 333-339.

30. Schouten JN, Garcia Pagan JC, Valla DC, et al. Idiopathic noncirrhotic portal hypertension. Hepatology. 2011;54(3):1071-1081. 
31. Saigal S, Nayak NC, Jain D. Noncirrhotic portal fibrosis related end stage liver disease in adults: evaluation from a study on living donor liver transplant recipients. Hepatol Int. 2011;5(4): 882-889.

32. Nayak NC, Jain D, Saigal S, et al.Non-cirrhotic portal fibrosis: one disease with many names? An analysis from morphological study of native explant livers with end stage chronic liver disease. J Clin Pathol. 2011;64(7): 592-598.

33. Bernard PH, Le Bail B, Cransac M, et al. Progression from idiopathic portal hypertension to incomplete septal cirrhosis with liver failure requiring liver transplantation. J Hepatol. 1995;22(4):495-499.

34. Stromeyer FW, Ishak KG. Nodular transformation (nodular "regenerative" hyperplasia) of the liver. A clinicopathologic study of 30 cases. Hum Pathol. 1981;12(1):60-71.

35. Elariny HA, Mizrahi SS, Hayes DH, et al. Nodular regenerative hyperplasia: a controversial indication for orthotopic liver transplantation. Transpl Int. 1964;7(4):309-313.

36. Radomski JS, Chojnacki KA, Moritz MJ, et al. Results of liver transplantation for nodular regenerative hyperplasia. Am Surg. 2000;66(11):1067-1070.
37. Loinaz C, Colina F, Musella M, et al. Orthotopic liver transplantation in 4 patients with portal hypertension and non-cirrhotic nodular liver. Hepatogastroenterology. 1998;45(23):1787-1794.

38. Dumortier J, Bizollon T, Scoazec JY, et al. Orthotopic liver transplantation for idiopathic portal hypertension: indications and outcome. Scand $J$ Gastroenterol. 2001;36(4):417-422.

39. Blanc JF, Bernard PH, Le Bail B. Vascular pathology of the portal vein distal branches: a rare cause of liver transplantation and a protean clinical presentation. Gastroenterol Clin Biol. 2000;24(6-7):667-670.

40. Pal S, Desai PR, Rao G. Non-cirrhotic portal fibrosis: results of surgery in 317 consecutive cases over a 25 -year period from an Indian center. Gastroenterology. 2002;123: T1454:88.

41. Krasinskas AM, Eghtesad B, Kamath PS, et al. Liver transplantation for severe intrahepatic noncirrhotic portal hypertension. Liver Transpl. 2005;11(6):627-634

42. Arun Kumar K, Arul Selvan V, Subramanian S, et al. Natural history of non cirrhotic portal hypertension-A prospective study. J Clin Experimental Hepatol. 2011;1(1):45. 